\title{
Ologen versus Mitomycin-C for Trabeculectomy in a Predominantly African American Population
}

\author{
Kenneth C Fan*, Howard Guan, Meenakshi Chaku, Babak Eliassi-Rad and Manishi A Desai
}

Department of Ophthalmology, Boston Medical Center/Boston University School of Medicine, Boston, MA 02118, USA

*Corresponding author: Kenneth C Fan, Department of Ophthalmology, Boston Medical Center/Boston University School of Medicine, Boston, MA 02118, USA, Tel: 2078077041; Fax: 617414929; E-mail: kennethcfan@gmail.com

Received date: May 20, 2016; Accepted date: July 08, 2016; Published date: July 12, 2016

Copyright: (c) 2016 Fan KC, et al. This is an open-access article distributed under the terms of the Creative Commons Attribution License, which permits unrestricted use, distribution, and reproduction in any medium, provided the original author and source are credited

\begin{abstract}
Objective: To investigate the efficacy and safety of the collagen-based Ologen implant as an adjuvant therapy in trabeculectomy as compared to mitomycin-C (MMC) in a predominantly African American patient population.

Methods: This study was a retrospective review of 54 eyes of 50 primary open angle glaucoma patients who underwent trabeculectomy with either MMC or Ologen. 16 eyes were included in the MMC group and 38 in the Ologen group. Primary outcomes included treatment success (as defined by target IOP $<21 \mathrm{mmHg}$, IOP reduction $>20$ ) both qualified (with and without medications) and unqualified (without medication), overall IOP reduction, and reduction of number of medications as compared to pre-operative state. Secondary outcomes included evaluation of treatment adverse events.
\end{abstract}

Results: No significant differences were noted between the two groups in comparing rates of qualified and unqualified success $(p=0.308$ and $p=0.343$, respectively). IOP reduction was seen for both groups at 3,6 , and 12 months, but no statistically significant differences were reached $(p=0.94,0.88$, and 0.84 , respectively). When examining medication reduction from pre-operative states, a significant difference in medication reduction was seen at the 6-month time point for the Ologen group as compared to the MMC group $(p=0.005)$. No significant differences were reached for the 3 or 12 -month time points $(p=0.051,0.341$, respectively). A significantly higher rate of postoperative bleb leak was noted with $\mathrm{MMC}$ as compared with Ologen $(p=0.009)$. No other adverse events showed differences between the two treatment groups.

Conclusion: Our findings suggest that Ologen is at least as effective as MMC in IOP reduction, reduction of postoperative medication use, and success rates when used as adjuvant therapy in trabeculectomy in a predominantly African American population. Ologen is also a safer option for patients of the same demographic demonstrated by lower rates of adverse events as compared to MMC.

Keywords: Ologen; Mitomycin-C; Trabeculectomy; African American; Adjuvant therapies in Trabeculectomy; Glaucoma

\section{Introduction}

Glaucoma is the most common cause of irreversible blindness worldwide [1]. Treatment of glaucoma begins with medical management but often requires surgical intervention. Since the late 1960s, the most common surgical treatment for glaucoma has been trabeculectomy [2-4]. AGIS investigators and others have established that race plays a significant role in an individual's response to trabeculectomy [5-7]. Specifically, African American patients have been shown to have advanced glaucoma at time of diagnosis and respond less favorably than Caucasian patients to trabeculectomy [5-14]. Our group wishes to investigate the role of ethnicity in specific surgical treatments for glaucoma.

Another issue pertaining to the failure of surgical intervention for glaucoma is post-operative wound scar formation resulting in fibrosis and obstruction of outflow, which remains the number one cause of failed trabeculectomies [15-17]. Mitomycin-C (MMC) is a commonly used intra-operative, anti-tumor metabolite that reduces postoperative fibrosis and scarring [18]. However, the existence of adverse effects of MMC, such as bleb leaks and avascular blebs [19], have led to the investigation of Ologen (Aeon Astron Europe B.V., Leiden, The Netherlands), a collagen glycosaminoglycan matrix implant, as another adjuvant therapy for trabeculectomy. In recent studies, some investigators have found that trabeculectomy with Ologen offers similar results as compared to MMC with no statistically significant difference in outcomes or complications between the two [20-24]. Others have found that Ologen offers less reduction in intra-ocular pressure (IOP), an increase in some complications, and lower complete and qualified success rates $[25,26]$. A more recent study looking at a longer term outcomes reported Ologen as significantly more successful than MMC in trabeculectomy patients [27].

No studies to this date have compared the efficacy of Ologen to MMC in African American patients. The fact remains that African American patients tend to have more progressed glaucoma at time of diagnosis and also have a lower rate of response to trabeculectomy. Given that current literature demonstrates conflicting evidence regarding the efficacy of trabeculectomy with MMC versus Ologen, the purpose of this study was to directly compare these two adjuvant therapies in a particularly vulnerable population of glaucoma patients. Our hypothesis was that trabeculectomy with Ologen would be at least 
Page 2 of 6

as effective as trabeculectomy with MMC in African American patients.

\section{Materials and Methods}

A retrospective review was performed by two independent reviewers in a sequential manner. All patients spanning between 2011 and 2013 who underwent trabeculectomy or combined trabeculectomy/phacoemulsification with either Ologen or MMC were included in this study. Other inclusion criteria were age 18 years or greater and current or past patient of Boston University Eye Associates. The protocol for this study was approved by the institutional review boards of Boston Medical Center.

Patient demographic and pre-operative data were collected by two independent reviewers. The demographic data were collected from clinical notes recorded from each patient visit. Medications taken by patients included both topical and oral glaucoma medications. The surgeries were performed by three experienced glaucoma surgeons $(\mathrm{BER} / \mathrm{MC} / \mathrm{MD})$. The majority of surgeries were performed with a fornix based peritomy. For MMC subjects, $0.4 \mathrm{mg} / \mathrm{ml}$ of MMC was applied for 2 to 2.5 minutes. For the Ologen group, an implant of $6 \times 2$ $\mathrm{mm}$ size was used.

The primary outcome measure was rate of treatment success. Other outcome measures included IOP reduction after surgery, reduction in the number of medications after surgery, and surgical complications. A success was defined as IOP <21 mmHg; and IOP reduction $>20 \%$; and required no repeat surgical intervention. An unqualified success was defined as success without medications, and a qualified success was defined by the same criteria with and without medication Complications evaluated included clinical identification of blebitis/ endophthalmitis, bleb leak, cataract, corneal ulcer, vein occlusion, dysesthesia, epithelial defect, hyphema, hypotony/choroidals, lens dislocation, rebound inflammation, retinal detachment, and tenon cysts. As this was a retrospective review, no bleb morphology staging was performed for analysis.

A total sample size of 54 eyes was used to examine the difference in efficacy between MMC and Ologen. The two groups were compared using chi-square test for categorical characteristics and unpaired Student's t-tests for continuous characteristics. Mean percent IOP reduction from baseline, and mean reduction in number of medications from baseline, and mean number and type of surgical complications were compared between the two groups across time. Surgical complications were compared using Fisher Exact $2 \times 2$ table tests. Distributions of time to loss of qualified and unqualified success were compared between the MMC and Ologen groups using the LogRank test. Estimates of rates of qualified and unqualified success were estimated using Kaplan-Meier methods and plotted over the course of 40 months. Statistical analyses were performed with SAS (version 9.3; SAS Institute Inc., Cary, NC, USA). Statistical significance was considered if $\mathrm{P}$-value $<0.05$.

\section{Results}

54 eyes from 50 consecutive patients with uncontrolled glaucoma undergoing trabeculectomy or combined trabeculectomy/ phacoemulsification with either Ologen or MMC were included in this study. Patient demographic data are summarized in Table 1.
Of 54 eyes, 16 received MMC and 38 received Ologen. 6 from the MMC group and 21 from the Ologen group underwent combined trabeculectomy/phacoemulsification.

\begin{tabular}{|c|c|c|c|c|}
\hline Characteristic & $\begin{array}{l}\text { Overall } \\
(\mathrm{N}=54)\end{array}$ & $\begin{array}{l}\text { MMC } \\
(\mathrm{N}=16)\end{array}$ & $\begin{array}{l}\text { Ologen } \\
(N=38)\end{array}$ & $\begin{array}{l}p- \\
\text { value }\end{array}$ \\
\hline Race & & & & 0.331 \\
\hline Black & $41(75.93 \%)$ & $12(75 \%)$ & $\begin{array}{l}29 \\
(76.32 \%)\end{array}$ & \\
\hline White & $5(9.26 \%)$ & $\begin{array}{l}3 \\
(18.75 \%)\end{array}$ & $2(5.26 \%)$ & \\
\hline Hispanic & $7(12.96 \%)$ & $1(6.25 \%)$ & $6(15.79 \%)$ & \\
\hline Asian & $1(1.85 \%)$ & $0(0.0 \%)$ & $1(2.63 \%)$ & \\
\hline Mean Age, years (SD) & $69.69(9.77)$ & $\begin{array}{l}67.94 \\
(7.81)\end{array}$ & $70.42(10.5)$ & 0.399 \\
\hline Diagnosis & & & & 0.002 \\
\hline POAG & $45(83.33 \%)$ & $\begin{array}{l}10 \\
(62.5 \%)\end{array}$ & $35(92.11 \%)$ & \\
\hline CACG & $4(7.41 \%)$ & $4(25 \%)$ & $0(0.0 \%)$ & \\
\hline MMG & $2(3.7 \%)$ & $0(0.0 \%)$ & $2(5.26 \%)$ & \\
\hline PDG & $2(3.7 \%)$ & $2(12.5 \%)$ & $0(0.0 \%)$ & \\
\hline Uveitic & $1(1.85 \%)$ & $0(0.0 \%)$ & $1(2.63 \%)$ & \\
\hline $\begin{array}{l}\text { Total Follow-up Time, months } \\
\text { (SD) }\end{array}$ & $17.5(9.5)$ & $\begin{array}{l}17.1 \\
(12.3)\end{array}$ & $17.7(8.24)$ & 0.853 \\
\hline Sex & & & & 0.694 \\
\hline Male & $19(35.19 \%)$ & $\begin{array}{l}5 \\
(31.25 \%)\end{array}$ & $\begin{array}{l}14 \\
(36.84 \%)\end{array}$ & \\
\hline Female & $35(64.81 \%)$ & $\begin{array}{l}11 \\
(68.75 \%)\end{array}$ & $\begin{array}{l}24 \\
(63.16 \%)\end{array}$ & \\
\hline Lens Type & & & & 0.833 \\
\hline NSC & $50(92.59 \%)$ & $\begin{array}{l}15 \\
(93.75 \%)\end{array}$ & $35(92.11 \%)$ & \\
\hline PCIOL & $4(7.41 \%)$ & $1(6.25 \%)$ & $3(7.89 \%)$ & \\
\hline Visual Acuity, Mean (SD) & $0.43(0.53)$ & $\begin{array}{l}0.47 \\
(0.69)\end{array}$ & $0.42(0.45)$ & 0.743 \\
\hline IOP, Mean (SD) & $21.31(7.34)$ & $21(7.6)$ & $21.45(7.33)$ & 0.840 \\
\hline $\begin{array}{l}\text { Number of Medications, Mean } \\
\text { (SD) }\end{array}$ & $3.2(1.28)$ & $2.81(1.6)$ & $3.37(1.1)$ & 0.147 \\
\hline \multicolumn{5}{|c|}{$\begin{array}{l}\text { IOP: Intra-Ocular Pressure; SD: Standard Deviation; POAG: Primary Open- } \\
\text { Angle Glaucoma; CACG: Chronic Angle Closure Glaucoma; MMG: Mixed } \\
\text { Mechanism Glaucoma; PDG: Pigment Dispersion Glaucoma; NSC: Nuclear } \\
\text { Sclerotic Cataract; PCIOL: Posterior Capsule Intra-Ocular Lens } \\
\text { Statistical significance determined by p-value }<0.05\end{array}$} \\
\hline
\end{tabular}

Table 1: Pre-Operative Patient Demographics

Overall, 41 subjects were African American; 12 were treated by MMC and 29 by Ologen resulting in similar proportions of African American patients in both groups. There was a significant difference in the proportion of patients with POAG between the two groups. 


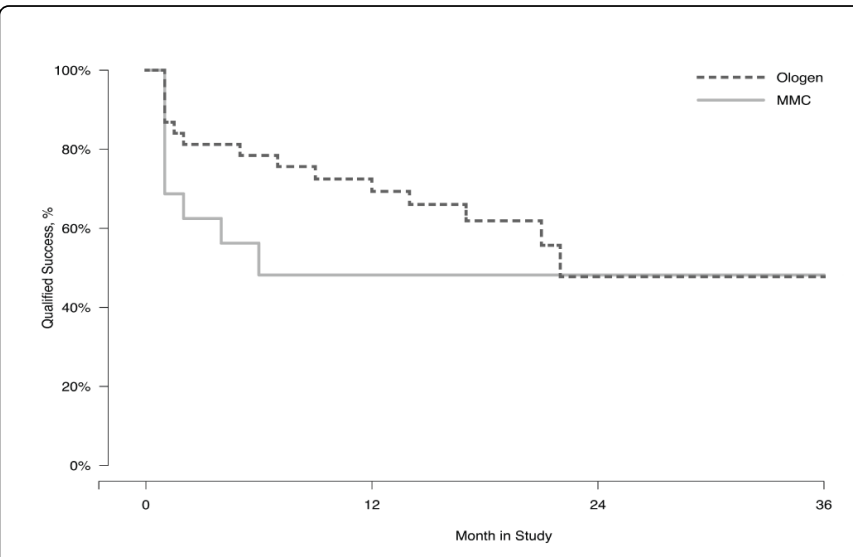

Figure 1: Kaplan-Meier Chart of Qualified Success Rates KaplanMeier chart demonstrating qualified success rates for both MMC (solid line) and Ologen (dotted line) groups. Qualified success was defined by reduction of IOP $<21 \mathrm{mmHg}$ and IOP reduction $>20 \%$ with no repeat surgical interventions, with and without medications. Patients both using and not using IOP lowering medications are included in the group of subjects who have achieved qualified success for treatment. Rates for success are noticeably higher until approximately the 24 month time point. However, no statistical significance was reached for the differences in qualified success rates between the two groups $(\mathrm{p}=0.308)$. At 12 months, qualified success rates were $72 \%$ and $47 \%$ for Ologen and $\mathrm{MMC}$, respectively.

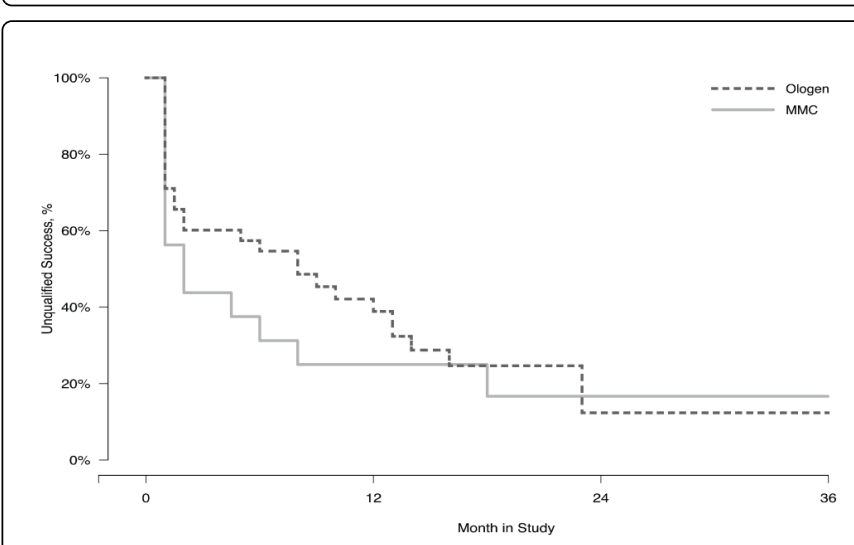

Figure 2: Kaplan-Meier Chart of Unqualified Success Rates KaplanMeier charts demonstrating unqualified success rates for both MMC (solid line) and Ologen (dotted line) groups. Unqualified success was defined by reduction of IOP $<21 \mathrm{mmHg}$ and IOP reduction $>20 \%$ with no repeat surgical interventions required. Additionally, to reach unqualified success, the patient could not be on any medications to reduce IOP. Rates for unqualified success are higher for the Ologen group until approximately the 18 month time point. Again, no statistical significance was reached for the differences in unqualified success rates between the two groups $(\mathrm{p}=0.343)$. At 12 months, unqualified success rates were $42 \%$ and $25 \%$ for Ologen and MMC, respectively.

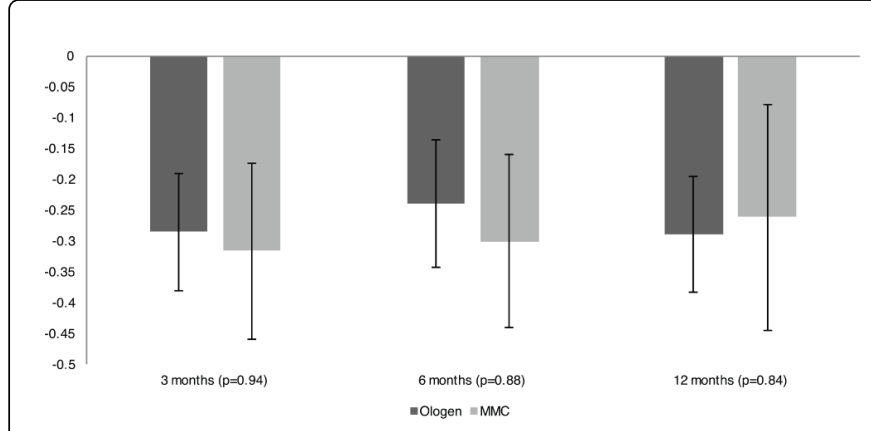

Figure 3: Post-Trabeculectomy IOP Reduction Histogram displaying percent IOP reduction at 3,6, and 12 month time points post-operatively in both Ologen (darker gray) and MMC (lighter gray) subject groups after trabeculectomy. P-values are shown for each respective time point. No statistically significant differences were observed as determined by $\mathrm{p}$-value $<0.05$.

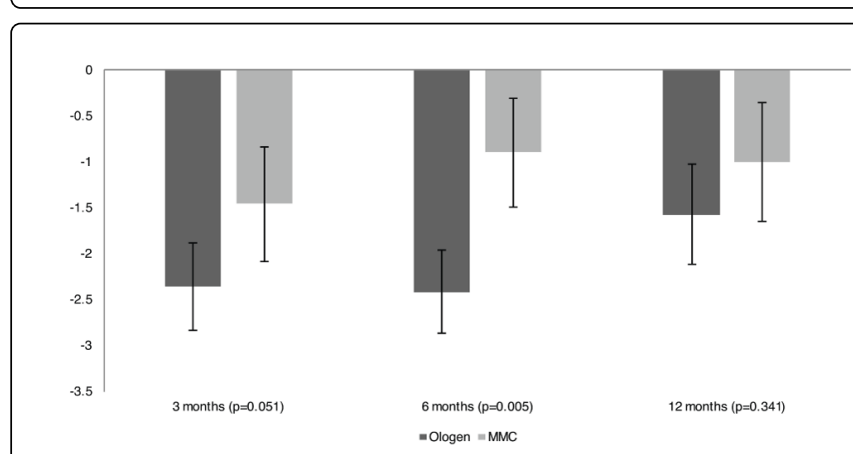

Figure 4: Post-Trabeculectomy Medication Reduction Histogram displaying reduction of number of post-operative medications at 3 , 6 , and 12 month time points in both Ologen (darker gray) and MMC (lighter gray) subject groups after trabeculectomy. Units are displayed in number of medications. P-values are shown for each respective time point. Statistical significance was reached for change in number of post-op medications at the 6 month time point as determined by $\mathrm{p}$-value $<0.05$.

Figure 1 and Figure 2 illustrate the Kaplan-Meier charts for both groups for qualified and unqualified success. In general, Ologen had higher qualified and unqualified success versus MMC at most timepoints. For qualified success, $\mathrm{p}=0.308$ and for the unqualified success, $\mathrm{p}=0.343$. Neither of these differences was statistically significant. At 12 months, qualified and unqualified success rates were $72 \%$ and $42 \%$ for Ologen, respectively. In the same time period, MMC qualified and unqualified success rates were $47 \%$ and $25 \%$, respectively.

Table 2 shows the tests for significance in the differences between the two tests groups at 3, 6, and 12 month time points for postoperative IOP reduction and medication reduction. IOP reduction was seen at 3, 6, and 12 months for both groups as seen in Figure 3, with no significant differences reached. However, greater medication reduction was seen in the Ologen group at 3,6, and 12 months as compared to MMC group as seen in Figure 4. Of these differences, medication reduction from pre-operative regimen at 6 months was significant $(\mathrm{p}=0.005)$. 


\begin{tabular}{|l|c|l|l|l|l|}
\hline & \multicolumn{2}{|l|}{ Ologen $(\mathrm{n}=38)$} & \multicolumn{2}{l|}{$\mathrm{MMC}(\mathrm{n}=16)$} & P-value \\
\hline & Mean & $95 \% \mathrm{Cl}$ & Mean & $95 \% \mathrm{Cl}$ & \\
\hline$\% \Delta$ IOP at 3 months & $-28 \%$ & $\pm 9.56 \%$ & $-32 \%$ & $\pm 14.3 \%$ & 0.940 \\
\hline$\% \Delta$ IOP at 6 months & $-24 \%$ & $\pm 10.2 \%$ & $-30 \%$ & $\pm 14.0 \%$ & 0.879 \\
\hline$\% \Delta$ IOP at 12 months & $-29 \%$ & $\pm 9.36 \%$ & $-26 \%$ & $\pm 18.2 \%$ & 0.841 \\
\hline & & & & & \\
\hline$\Delta$ in \# Meds at 3 months & -2.4 & \pm 0.475 & -1.5 & \pm 0.626 & 0.051 \\
\hline$\Delta$ in \# Meds at 6 months & -2.4 & \pm 0.446 & -0.9 & \pm 0.598 & 0.005 \\
\hline$\Delta$ in \# Meds at 12 months & -1.6 & \pm 0.540 & -1.0 & \pm 0.642 & 0.341 \\
\hline
\end{tabular}

Cl: Confidence Interval; IOP: Intra-Ocular Pressure

$\Delta$ in IOP expressed in \% change

$\Delta$ in number of medications denoted by number of medications

Statistical significance determined by $p$-value $<0.05$

Table 2: Post-operative Change in Intra-ocular Pressure and Number of Medications

\begin{tabular}{|c|c|c|c|c|}
\hline Characteristic & $\begin{array}{l}\text { Overall } \\
(\mathrm{N}=54)\end{array}$ & $\begin{array}{l}\mathrm{MMC} \\
(\mathrm{N}=16)\end{array}$ & $\begin{array}{l}\text { Ologen } \\
(\mathrm{N}=38)\end{array}$ & $p$-value \\
\hline Blebitis & $4(7.41 \%)$ & $2(12.5 \%)$ & $2(5.26 \%)$ & 0.354 \\
\hline Bleb Leak & $14(25.93 \%)$ & $8(50 \%)$ & $6(15.79 \%)$ & 0.009 \\
\hline $\begin{array}{l}\text { Bleb Leak requiring re- } \\
\text { operation }\end{array}$ & $3(5.56 \%)$ & $2(12.5 \%)$ & $1(2.63 \%)$ & 0.148 \\
\hline Corneal Ulcer & $1(1.85 \%)$ & $1(6.25 \%)$ & $0(0.0 \%)$ & 0.120 \\
\hline CRVO & $1(1.85 \%)$ & $0(0.0 \%)$ & $1(2.63 \%)$ & 0.512 \\
\hline Dysesthesia & $5(9.26 \%)$ & $\begin{array}{l}3 \\
(18.75 \%)\end{array}$ & $2(5.26 \%)$ & 0.118 \\
\hline Endophthalmitis & $1(1.85 \%)$ & $0(0.0 \%)$ & $1(2.63 \%)$ & 0.512 \\
\hline Epithelial Defect & $2(3.7 \%)$ & $0(0.0 \%)$ & $2(5.26 \%)$ & 0.350 \\
\hline Hyphema & $2(3.7 \%)$ & $1(6.25 \%)$ & $1(2.63 \%)$ & 0.520 \\
\hline Hypotony & $3(5.56 \%)$ & $1(6.25 \%)$ & $2(5.26 \%)$ & 0.885 \\
\hline Hypotony with Choroidals & $3(5.56 \%)$ & $2(12.5 \%)$ & $1(2.63 \%)$ & 0.148 \\
\hline IOL Dislocation & $1(1.85 \%)$ & $1(6.25 \%)$ & $0(0.0 \%)$ & 0.120 \\
\hline Persistent Inflammation & $7(12.96 \%)$ & $0(0.0 \%)$ & $7(18.42 \%)$ & 0.066 \\
\hline Retinal Detachment & $1(1.85 \%)$ & $1(6.25 \%)$ & $0(0.0 \%)$ & 0.120 \\
\hline Tenons cyst & $1(1.85 \%)$ & $0(0.0 \%)$ & $1(2.63 \%)$ & 0.512 \\
\hline \multicolumn{5}{|l|}{$\begin{array}{l}\text { CRVO = Central Retinal } \\
\text { Vein Occlusion }\end{array}$} \\
\hline \multicolumn{5}{|l|}{$\mathrm{IOL}=$ Intra-ocular Lens } \\
\hline \multicolumn{5}{|c|}{$\begin{array}{l}\text { Persistent Inflammation defined as post-operative iritis } \geq 2 \text { weeks after } \\
\text { trabeculectomy }\end{array}$} \\
\hline $\begin{array}{l}\text { Statistical significance dete } \\
\text { value }<0.05\end{array}$ & rmined by $\mathrm{p}-$ & & & \\
\hline
\end{tabular}

Table 3: Post-operative Rate of Complications.
Table 3 displays the data regarding the rate of complications for each group. The most common post-operative complication across the entire cohort was bleb leakage (26\%). A significant difference was noted for bleb leaks between the treatment groups with the rate of leaks in MMC versus Ologen group being $50 \%$ vs. $16 \%, \mathrm{p}=0.009$ ), seen in Table 2. There was no significant difference in any of the remaining complications, however the rate of persistent inflammation in Ologen subjects was noted to be higher than in MMC subjects, but did not reach statistical significance $(\mathrm{p}=0.066)$.

\section{Discussion}

Glaucoma is the leading cause of irreversible blindness in the world today $[1,28]$. It is estimated that $3 \%$ of the global population over age 40 years old is affected by glaucoma, with many of these individuals unaware of their own conditions [28]. The most common surgical therapy for glaucoma is trabeculectomy [4]. Trabeculectomy remains the standard of care for surgical intervention with current success rates ranging from 52-95\% [29]. Adjuvant therapies, such as MMC or Ologen, have been used to reduce the rate of post-operative failure secondary to fibrosis and scar healing of the wound [30]. In African American patients with glaucoma, however, trabeculectomy has higher rates of failure as compared to Caucasian patients [5-14]. No studies have investigated the efficacy of trabeculectomy with Ologen versus MMC in a predominantly African American population, despite proven higher failure rates.

Ologen is a collagen implant that is placed atop the scleral flap prior to closure of the conjunctiva during trabeculectomy. Ologen encourages filtration of aqueous humor by allowing for absorption of the fluid into the porous structure of the collagen. Furthermore, Ologen discourages organized growth of fibroblasts in the wound healing process, resulting in lower rates of scar formation and failure of trabeculectomy surgery [31,32].

\section{Success Rates}

In our study, we have provided useful evidence of the efficacy of Ologen versus MMC when used in trabeculectomy in African American patients. In our subject groups, there were no demographic differences that predisposed either group to specific outcomes. We found that, similar to a recent study by Cillino et. al., [33] the Ologen group fared at least as well as the MMC group when comparing qualified and unqualified success rates at all time points (Figure 1 \& Figure 2). Though the differences were not statistically significant, the Kaplan-Meier curves do demonstrate a mild trend towards improved survival rates for the Ologen subjects. At 1 year post-op, the Ologen group demonstrated a $70 \%$ vs. $42 \%$ rate of qualified success and $40 \%$ vs. $8.3 \%$ rate of unqualified success compared to the MMC group. Though non-significant, these findings are in agreement with a study by Wang et al., who also found higher rates of success for Ologen. Our findings, however, do conflict with findings by Rosentreter et al. [34] and Narayanaswamy et al. [25] who found Ologen to be less successful when compared to MMC. The differences in our results may be due to the response to Ologen in our patient demographic. It has been well documented that African Americans have a decreased response to filtration surgery because of the increased number of macrophages and fibroblasts in their ocular tissues [8,9]. Because of increased fibroblastic proliferation and wound healing response in this demographic, collagenous structural barrier may modulate wound healing by acting as a scaffold for fibroblast regrowth, and may allow Ologen to increase rates of success as compared to MMC in 
trabeculectomy. Our hope is that future investigations with larger cohorts of African American patients and a specific focus on histopathology as well as bleb morphology will establish the difference in efficacy when comparing Ologen to MMC.

\section{Post-Operative Medication Reduction and IOP Reduction}

We also found that both groups experienced a reduction in postoperative IOP and number of medications from pre-operative levels, as previously established by other studies [20,26,27,34]. However, our Ologen subjects saw a significant reduction of number of postoperative medications at 6 months $(\mathrm{p}=0.021)$ as compared to MMC subjects, although other time points were not found to be significantly different. This indicates that Ologen is more effective than MMC in reducing post-operative topical and oral medications in a predominantly African American population at 6 months post-op. In a meta-analysis by $\mathrm{He}$ et al. and a prospective trial by Rosentreter, however, no significant differences were found between the two groups for medication reduction [21,26]. Due to our study demographics, generalized findings in these meta-analyses cannot be applied to our specific patient population.

We did not see statistically significant differences in IOP reduction between the groups ( $p=0.271,0.497,0.100$ at 3 months, 6 months, and 1 year, respectively). He et al. found that IOP reduction for patients who received Ologen was lower than MMC, but without any significant differences [21]. Cillino et al. also found that there was no significant difference between the two. These data are congruent with our study findings. In contrast, Narayanaswamy et al. has established that Ologen has less IOP reduction than MMC, but this study investigated Asian subjects, unlike our patient demographic. Although we did not look at IOP reduction stratified by diagnosis, Narayanaswamy et al. found that Ologen induced greater response in patients with POAG as compared to primary angle-closure glaucoma, where MMC had a greater effect [25]. This may have played a factor in our study, as most of our patients were diagnosed with POAG (83\%), but further studies looking at the effect of Ologen in different types of glaucoma may potentially reveal additional findings of interest.

\section{Complications and Adverse Events}

When examining rates of complications, the rate of post-operative bleb leak was significantly higher in the MMC group (50\%) vs. the Ologen group $(7.1 \%, p=0.002)$, with all other surgical complications found to have no statistically significant difference. Prior investigations have determined the range of bleb leakage rate for MMC to be $5-30 \%$ ( $\mathrm{n}=19$ to 227 patients) $[20,21,26,27]$. The same studies determined a bleb leakage rate for Ologen to be $3-30 \%$. Two previous investigators have established that usage of MMC results in more avascular blebs post-operatively because of its anti-metabolite effects $[25,26]$. Avascular blebs are well-known to be a risk factor for bleb leakage because of the thinning of the conjunctiva, marked loss of conjunctival epithelium, and reduction of goblet cells and thus protective mucin formation [35,36]. Our findings are therefore consistent with previous studies and we believe that Ologen can actually decrease the risk for complications after trabeculectomy as compared to MMC, especially for bleb leakage. This makes it a safe alternative to MMC in the use of trabeculectomies in a predominantly African American population.

Our retrospective review study has some limitations that must be considered. The main limitation is the retrospective nature of the study, which leaves the potential for bias from the investigator as well as exclusion of cases lost to follow-up. However, our selection of cases was as thorough as possible, with an inclusion of all trabeculectomy patients from 2011 to 2013 . We also were limited in our sample size and did not stratify for severity of glaucoma, which may have provided further insight in the efficacy of trabeculectomy in this target population. Being a retrospective study, post-operative visits were not standardized; therefore, the exact times of follow-up visits were not uniform. There were three total surgeons involved in this study, allowing for some variability in surgical technique. We did not investigate significant differences in outcomes between the three surgeons. However, the fundamental methodology did not vary from surgeon to surgeon. Small differences included usage of releasable and simple interrupted flap sutures and shapes of scleral flaps. We used traditional interrupted sutures for MMC. However, the opacity of the Ologen implant can obstruct the view for laser suture-lysis and therefore necessitated the use of releasable sutures. However, we do not believe this impacted the outcomes of our study as previous studies have indicated no significant differences between use of releasable sutures versus traditional flap sutures $[37,38]$. The blebs in our study were not graded morphologically due to the retrospective nature of the study. Furthermore, the timing of bleb leaks for our patient population was variable and requires further investigation to identify if the leaks were secondary to avascularization or poor peri-operative wound healing. Lastly, it is important to note that our study was performed on a predominantly African American patient population and cannot be generalized to other cohorts with differing demographics.

In conclusion, our data illustrates that overall performance of trabeculectomy with Ologen is at least as effective as MMC as a surgical intervention for adult glaucoma in African American patients. It is also a safe alternative, with fewer complications rates due to its lower rate of post-operative bleb leakage. We realize that additional studies with standardized protocols may be necessary in order to further explore the long-term efficacy and safety of Ologen in this population and hope to expand our study to draw more conclusive results in future investigations.

\section{References}

1. Quigley HA, Broman AT (2006) The number of people with glaucoma worldwide in 2010 and 2020. Br J Ophthalmol 90: 262-267.

2. Bevin TH, Molteno AC, Herbison P (2008) Otago Glaucoma Surgery Outcome Study: long-term results of 841 trabeculectomies. Clin Experiment Ophthalmol 36: 731-737.

3. Lockwood A, Brocchini S, Khaw PT (2013) New developments in the pharmacological modulation of wound healing after glaucoma filtration surgery. Curr Opin Pharmacol 13: 65-71.

4. Razeghinejad MR, Fudemberg SJ, Spaeth GL (2012) The changing conceptual basis of trabeculectomy: a review of past and current surgical techniques. Surv Ophthalmol 57: 1-25.

5. [No authors listed] (1998) The Advanced Glaucoma Intervention Study (AGIS): 4. Comparison of treatment outcomes within race. Seven-year results.Ophthalmology 105: 1146-1164.

6. AGIS Investigators (2001) The Advanced Glaucoma Intervention Study (AGIS): 9. Comparison of glaucoma outcomes in black and white patients within treatment groups. Am J Ophthalmol 132: 311-320.

7. Husain R, Clarke JC, Seah SK, Khaw PT (2005) A review of trabeculectomy in East Asian people--the influence of race. Eye (Lond) 19: 243-252.

8. Broadway D, Grierson I, Hitchings R (1994) Racial differences in the results of glaucoma filtration surgery: are racial differences in the conjunctival cell profile important? Br J Ophthalmol 78: 466-475. 
9. Broadway DC, Chang LP (2001) Trabeculectomy, risk factors for failure and the preoperative state of the conjunctiva. J Glaucoma 10: 237-249.

10. Tielsch JM, Sommer A, Katz J, Royall RM, Quigley HA, et al. (1991) Racial variations in the prevalence of primary open-angle glaucoma. The Baltimore Eye Survey. JAMA 266: 369-374.

11. Wilson R, Richardson TM, Hertzmark E, Grant WM (1985) Race as a risk factor for progressive glaucomatous damage. Ann Ophthalmol 17: 653-659.

12. Martin MJ, Sommer A, Gold EB, Diamond EL (1985) Race and primary open-angle glaucoma. Am J Ophthalmol 99: 383-387.

13. Wilensky JT, Gandhi N, Pan T (1978) Racial influences in open-angle glaucoma. Ann Ophthalmol 10: 1398-1402.

14. [No authors listed] (1998) The Advanced Glaucoma Intervention Study (AGIS): 3. Baseline characteristics of black and white patients. Ophthalmology 105: 1137-1145.

15. Addicks EM, Quigley HA, Green WR, Robin AL (1983) Histologic characteristics of filtering blebs in glaucomatous eyes. Arch Ophthalmol 101: 795-798.

16. Maumenee AE (1960) External filtering operations for glaucoma: the mechanism of function and failure. Trans Am Ophthalmol Soc 58: 319-328.

17. Skuta GL, Parrish RK 2nd (1987) Wound healing in glaucoma filtering surgery. Surv Ophthalmol 32: 149-170.

18. Lin ZJ, Li Y, Cheng JW, Lu XH (2012) Intraoperative mitomycin C versus intraoperative 5-fluorouracil for trabeculectomy: a systematic review and meta-analysis. J Ocul Pharmacol Ther 28: 166-173.

19. Seibold LK, Sherwood MB, Kahook MY (2012) Wound modulation after filtration surgery. Surv Ophthalmol 57: 530-550.

20. Cillino S, Di Pace F, Cillino G, Casuccio A (2011) Biodegradable collagen matrix implant vs mitomycin-C as an adjuvant in trabeculectomy: a 24 month, randomized clinical trial. Eye (Lond) 25: 1598-1606.

21. He M, Wang W, Zhang X, Huang W (2014) Ologen implant versus mitomycin $\mathrm{C}$ for trabeculectomy: a systematic review and meta-analysis. PLoS One 9: e85782.

22. Ji Q, Qi B, Liu L, Guo X, Zhong J (2015) Efficacy and Safety of Ologen Implant Versus Mitomycin C in Primary Trabeculectomy: A Metaanalysis of Randomized Clinical Trials. J Glaucoma 24: e88-94.

23. Johnson MS, Sarkisian SR (2014) Using a collagen matrix implant (Ologen) versus mitomycin-C as a wound healing modulator in trabeculectomy with the Ex-PRESS mini glaucoma device: a 12-month retrospective review. J Glaucoma 23: 649-652.

24. Marey HM, Mandour SS, Ellakwa AF (2013) Subscleral trabeculectomy with mitomycin-C versus ologen for treatment of glaucoma. J Ocul Pharmacol Ther 29: 330-334.

25. Narayanaswamy A, Perera SA, Htoon HM, Hoh ST, Seah SK, et al. (2013) Efficacy and safety of collagen matrix implants in phacotrabeculectomy and comparison with mitomycin $\mathrm{C}$ augmented phacotrabeculectomy at 1 year. Clin Experiment Ophthalmol 41: 552-560.

26. Rosentreter A, Schild AM, Jordan JF, Krieglstein GK, Dietlein TS (2010) A prospective randomised trial of trabeculectomy using mitomycin $\mathrm{C}$ vs an ologen implant in open angle glaucoma. Eye (Lond) 24: 1449-1457.

27. Yuan F, Li L, Chen X, Yan X, Wang L (2015) Biodegradable 3D-Porous Collagen Matrix (Ologen) Compared with Mitomycin C for Treatment of Primary Open-Angle Glaucoma: Results at 5 Years. J Ophthalmol 2015: 637537.

28. Varma R, Lee PP, Goldberg I, Kotak S (2011) An assessment of the health and economic burdens of glaucoma. Am J Ophthalmol 152: 515-522.

29. Sihota R, Gupta V, Agarwal HC (2004) Long-term evaluation of trabeculectomy in primary open angle glaucoma and chronic primary angle closure glaucoma in an Asian population. Clin Experiment Ophthalmol 32: 23-28.

30. Lee CH, Singla A, Lee Y (2001) Biomedical applications of collagen. Int J Pharm 221: 1-22.

31. Boey PY, Narayanaswamy A, Zheng C, Perera SA, Htoon HM, et al. (2011) Imaging of blebs after phacotrabeculectomy with Ologen collagen matrix implants. Br J Ophthalmol 95: 340-344.

32. Hsu WC, Spilker MH, Yannas IV, Rubin PA (2000) Inhibition of conjunctival scarring and contraction by a porous collagenglycosaminoglycan implant. Invest Ophthalmol Vis Sci 41: 2404-2411.

33. Cillino S, Casuccio A, Di Pace F, Cagini C, Ferraro LL, et al. (2016) Biodegradable collagen matrix implant versus mitomycin-C in trabeculectomy: five-year follow-up. BMC Ophthalmol 16: 24.

34. Rosentreter A, Gaki S, Cursiefen C, Dietlein TS (2014) Trabeculectomy using mitomycin $\mathrm{C}$ versus an atelocollagen implant: clinical results of a randomized trial and histopathologic findings. Ophthalmologica 231: $133-140$.

35. Lehmann OJ, Bunce C, Matheson MM, Maurino V, Khaw PT, et al. (2000) Risk factors for development of post-trabeculectomy endophthalmitis. Br J Ophthalmol 84: 1349-1353.

36. Nuyts RM, Felten PC, Pels E, Langerhorst CT, Geijssen HC, et al. (1994) Histopathologic effects of mitomycin C after trabeculectomy in human glaucomatous eyes with persistent hypotony. Am J Ophthalmol 118: 225-237.

37. Kobayashi H, Kobayashi K (2011) A comparison of the intraocular pressure lowering effect of adjustable suture versus laser suture lysis for trabeculectomy. J Glaucoma 20: 228-233.

38. Simsek T, Citirik M, Batman A, Mutevelli S, Zilelioglu O (2005) Efficacy and complications of releasable suture trabeculectomy and standard trabeculectomy. Int Ophthalmol 26: 9-14. 